\title{
A Micromachined Flow Shear-Stress Sensor Based on Thermal Transfer Principles
}

\author{
Chang Liu, Member, IEEE, Jin-Biao Huang, Zhenjun (Alex) Zhu, \\ Fukang Jiang, Steve Tung, Yu-Chong Tai, and Chih-Ming Ho
}

\begin{abstract}
Microhot-film shear-stress sensors have been developed by using surface micromachining techniques. The sensor consists of a suspended silicon-nitride diaphragm located on top of a vacuum-sealed cavity. A heating and heat-sensing element, made of polycrystalline silicon material, resides on top of the diaphragm. The underlying vacuum cavity greatly reduces conductive heat loss to the substrate and therefore increases the sensitivity of the sensor. Testing of the sensor has been conducted in a wind tunnel under three operation modes - constant current, constant voltage, and constant temperature. Under the constanttemperature mode, a typical shear-stress sensor exhibits a time constant of $72 \mu \mathrm{s}$. [362]
\end{abstract}

\section{INTRODUCTION}

$\mathbf{T}$ THE DETERMINATION of wall shear stress $\tau$ is very important in fluid mechanics and aerodynamics studies. For example, the viscous drag experienced by an object in a flow field corresponds to the integrated shear stress over the surface area. The ability of measuring the distribution of shear stress with high spatial and temporal resolution is critical for understanding and potentially controlling wallbound turbulence and flow separation.

Techniques for measuring the shear stress can be categorized into indirect and direct methods [1], [2]. Direct measurement relies on detection of the total amount of viscous drag experienced by a surface-mounted force balance. The drag force represents the area integration of the shear force. In order to obtain high sensitivity, it is desirable to have large surface areas in order to increase the amount of drag force and induced displacement.

In indirect methods, the shear stress is extracted from other measured physical parameters (e.g., pressure and wall temperature) that are related to the shear stress. For example, stream-wise distribution of pressure along a flow channel can be used to derive $\tau$ : this serves as the foundation of some well-established fluid mechanics calibration devices.

Manuscript received July 3, 1998; revised November 18, 1998. Subject Editor, G. Stemme.

C. Liu and Z. A. Zhu are the Microelectronics Laboratory, University of Illinois, Urbana, IL 61801 USA (e-mail: changliu@uiuc.edu).

F. Jiang and Y.-C. Tai are with the Electrical Engineering Department, California Institute of Technology, Pasadena, CA 91125 USA.

J.-B. Huang, S. Tung, and C.-M. Ho are with the Mechanical, Aeronautics, and Nuclear Engineering Department, University of California, Los Angeles, CA 90007 USA.

Publisher Item Identifier S 1057-7157(99)02056-9.
Variations of Pitot tubes, ${ }^{1}$ such as Preston tubes [2], [3] and Stanton tubes [4], [5], have been used for measurements of this nature. However, there are many disadvantages to these types of devices-ports for pressure measurements require modification to the wall and present potential disturbance to the flow.

Flow measurements based on thermal transfer principles is an indirect method and have been widely used [1]. Examples include flow-rate sensors with hot-wire or hot-film configurations. Compared with Pitot-tube measurement techniques, thermal sensors can be used in a wide variety of flow conditions and present minimal disturbance to the flow itself because of the surface flush mount.

The availability and performance of flow sensors have been seriously limited by traditional fabrication techniques. It is difficult to miniaturize conventional sensors because their manufacturing typically requires delicate handicraft. For the same reason, mass production with good device repeatability was very challenging. Micromachined sensors, on the other hand, can realize much reduced sizes. It is also easy to produce large quantities of sensors with uniform geometry and performance. In recent years, both indirect and direct shearstress sensors have been implemented using micromachining technology. For example, surface floating-element microbalances for direct shear-stress measurements have been reported [6]-[8]. The measured sensitivity was $52 \mu \mathrm{V}(\mathrm{ac}) / \mathrm{Pa}$ in a gaseous medium using a differential capacitor readout scheme and $13.7 \mu \mathrm{V} / \mathrm{V}-\mathrm{kPa}$ in a liquid medium using a piezoresistive readout scheme. Recently, integrated photodiodes have been used to sense displacement [8], realizing a sensitivity of $0.1 \% / \mathrm{Pa}$.

We have developed micromachined shear-stress sensors based on thermal transfer principles. The design, fabrication, and testing of such sensors, which contains novel thermal isolation structures, are presented in this paper.

\section{OPERATING PRINCIPLES}

The operation principle of a thermal shear-stress sensor is briefly described below. The sensor consists of a thermal element located on the surface of a substrate. The thermal element resides with a velocity boundary layer in which the velocity changes from zero (at the wall) to the value of the mean-stress flow. The rate of heat loss from a heated resistive

\footnotetext{
${ }^{1}$ A flow pressure measurement device with one or multiple pressure-input ports. The device must extend into the flow region to measure local pressure; this device will therefore create disturbance to the flow field.
} 


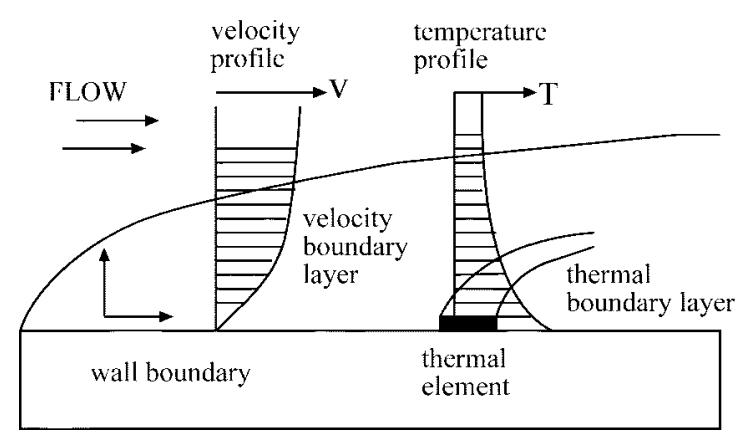

Fig. 1. Velocity and temperature boundary layers.

element to the air flow is dependent on the velocity profile in the boundary layer. The shear stress is expressed as

$$
\tau=\mu \frac{d}{d y} U_{y}
$$

where $\mu$ is the viscosity of the fluid and $U_{y}$ is the flow velocity at a distance $y$ from the wall. As a result, the flow shear stress determines the rate of heat transfer from the heated element to the surrounding flow field. The temperature change of the resistor can be measured by the change in its resistance. The resistance $(R)$ of a semiconductor sensing element at an elevated temperature $T$ is expressed as

$$
R=R_{0}\left(1+\alpha\left(T-T_{0}\right)\right)
$$

where $R_{0}$ is the resistance at the room temperature $T_{0}$ and $\alpha$ is the temperature coefficient of resistance often termed TCR.

A thermal boundary layer is characterized as a region where temperature gradients are present in the flow. Typical profiles of thermal and velocity boundary layers are illustrated in Fig. 1 for the case of a two-dimensional (2-D) heat generating/sensing element $\left(\right.$ area $=A_{e}$ ) inside of a 2-D channel flow. Within the thermal boundary layer, flow temperature decreases with increasing distance away from the heated element until the temperature reaches that of the mean stream flow.

The relationship between $\tau$ and the temperature of the heated resistor has been studied [9] under a major assumption that the thermal boundary layer of the thermal element lies within the velocity boundary layer. Under several other assumptions, the relationship between the input power, shear stress at the wall, and temperature of the thermal element can be expressed in simply analytical form. These assumptions include: 1) the thermal transfer in the span-wise direction can be ignored and 2) effects of natural convection are much smaller compared with forced convection. Under ohmic heating, the input power is related to the driving current and the sensor resistance. Since part of the input power is transferred to the flow while the rest is lost through the substrate, the power balance is expressed as

$$
i^{2} R=\triangle T\left(A(\rho \tau)^{1 / 3}+B\right)
$$

where $A$ equals $0.807 A_{e} C_{p}^{1 / 3} k_{T}^{1 / 3} / L^{1 / 3} \mu^{1 / 3}$ and $B$ signifies the conduction heat loss to the substrate. Here, $A_{e}$ is the effective area of the thermal element, $C_{p}$ is the heat capacity of the flow, $k_{T}$ is the thermal conductivity of the fluid, $L$ is the stream-wise length of the resistor, and $\mu$ is the viscosity. At a certain input power $i^{2} R$, the greater the term $B$ is compared with $A \tau^{1 / 3}$, the smaller the shear-stress sensitivity will be. Therefore, to design a successful shear-stress sensor, the heat loss to the substrate must be minimized.

Thermal shear-stress sensors realized using traditional fabrication technology suffer from two major drawbacks. Because metal (e.g., nickel and platinum) is routinely used as the heating and sensing element, the resistance of sensing element is low and a large biasing current is typically required to produce adequate surface-heating effects. Second, the sensitivity of such devices is low because there is no effective heat insulation between the heated element and the substrate, due to difficulties of fabrication and packaging.

The current status of microthermal flow sensors is reviewed in the following. There have been only a few published reports on micromachined shear-stress sensors [10], [11]. In [10], a pressure sensor and flow sensor are integrated for measurements in turbulent gas flows. Thermal piles are used in [11] to detect temperature changes. In addition to developed shearstress sensors, microfabricated flow sensors have been realized by several groups [12]-[14]. The principle and construction of thermal shear-stress sensors is comparable with thermal flow sensors. In recent years, microflow sensors have been demonstrated using both bulk and surface micromachining technologies.

Microsensors present device-performance improvement in two ways. First, the semiconductor material employed as the heating/sensing element has a much higher resistivity compared to metal. A small current is adequate in generated required heating effects. Second, unique heat insulation features can be realized to solve the common, but major problem of the heat loss to the substrate, which limits the performance of conventional thermal shear-stress sensors. Such thermal-isolation features include freestanding beams [15], [16], freestanding diaphragms [17], and low thermal-conductivity layer such as polyimide [18], [19].

\section{SENSOR DESIGN}

Fig. 2 shows the schematic top and side views of a shearstress sensor. The heating and heat-sensing element is a resistor made of phosphorous-doped polysilicon material. The resistors are $2 \mu \mathrm{m}$ wide and $0.45 \mu \mathrm{m}$ thick, and their length ranges from 20 to $200 \mu \mathrm{m}$. The resistors are uniformly doped to a low-sheet-resistance value of $50 \Omega / \square$ with typical resistances between $1.25-5 \mathrm{k} \Omega$ at the room temperature for the range of resistor lengths indicated above. Each resistor is located at the center of a cavity diaphragm, which is typically $200 \times$ $200 \mu \mathrm{m}^{2}$ in area and $1.5 \mu \mathrm{m}$ in thickness. The diaphragm is separated from the bottom of the cavity by approximately 2 $\mu \mathrm{m}$, with the pressure inside the cavity being lower than 300 mtorr. Two metallization wires, each $10 \mu \mathrm{m}$ wide, connect the polysilicon resistor to the external electronics.

The novel aspect of the sensor is that the diaphragm lies on top of a vacuum cavity, which minimizes the heat conduction from the diaphragm to the substrate through the gap; this feature offers effective thermal isolation between the heated element and the substrate. In the current design, the depth of 


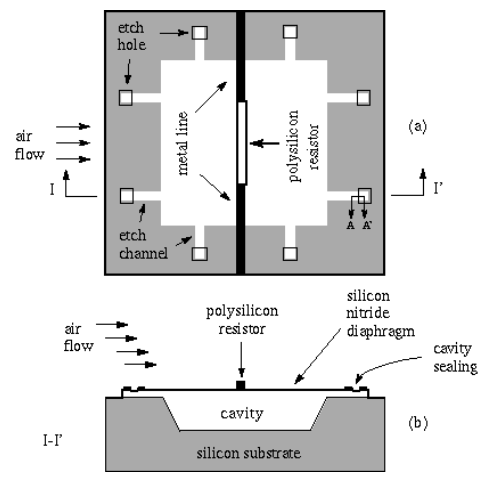

Fig. 2. Schematic top and side views of a thermal shear-stress sensor.

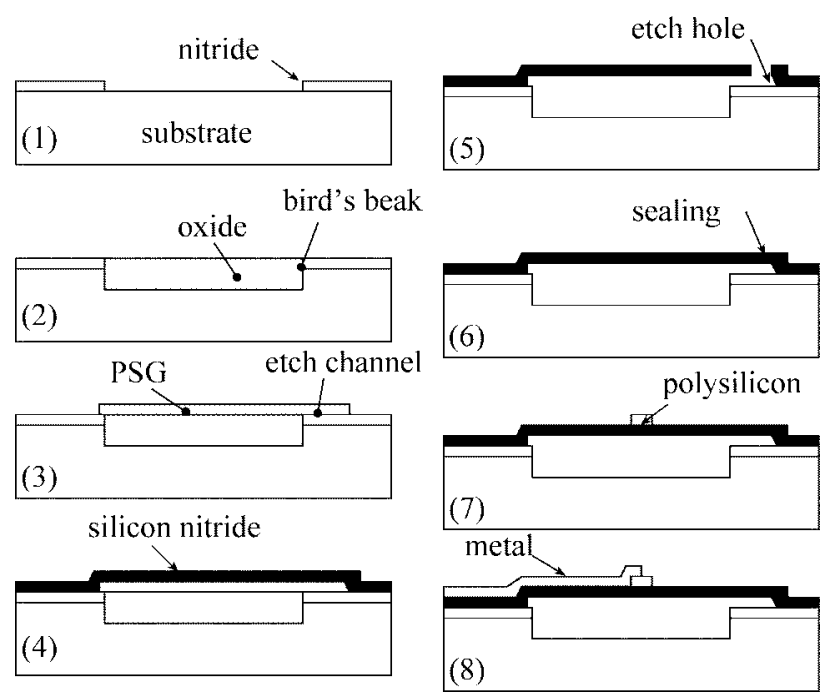

Fig. 3. Fabrication steps of a shear-stress sensor.

the gap is $2 \mu \mathrm{m}$. The heat isolation can be further improved by increasing the depth of the cavity, thus enhancing the thermal resistance from the diaphragm to the substrate. However, this would make the fabrication process more complex. For example, since the thickness of the regrown thermal oxide corresponds to the depth of the cavity, it would require a longer time for oxide growth in order to form a deeper cavity.

\section{SENSOR FABRICATION}

The micromachining fabrication process is illustrated in Fig. 3. We start the process with a 4-in-diameter silicon wafer. First, a $0.4-\mu \mathrm{m}$ silicon nitride layer is deposited by low-pressure chemical vapor deposition (LPCVD) and photolithographically patterned to define location and shape of the cavities. Each future cavity is defined as a $200 \times 200$ $\mu \mathrm{m}^{2}$ square window in which the silicon nitride material is removed using SF6 plasma etching, exposing the underlying silicon substrate.

The ideal depth of each cavity, measured from the silicon nitride surface to the bottom of the silicon surface, is 0.7 $\mu \mathrm{m}$. A $1.3-\mu \mathrm{m}$-thick silicon dioxide is grown using thermal oxidation at $1050^{\circ} \mathrm{C}$ in $4 \mathrm{~h}$. During the oxidation process, the silicon/silicon dioxide interface moves further into the substrate. At the end of this oxidation process, $44 \%$ of the

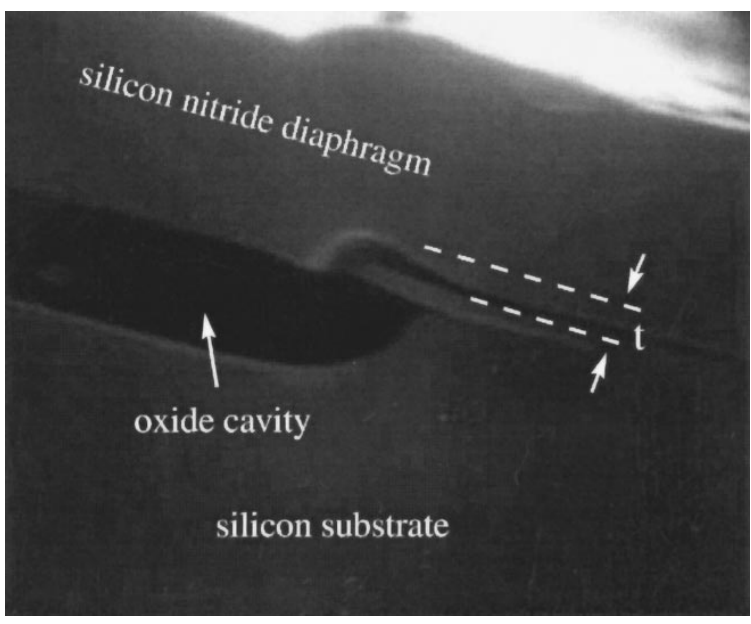

Fig. 4. Bird's beak structure related to microcavities.

1.3- $\mu \mathrm{m}$ oxide thickness will be contributed by oxidation below the original silicon surface [20].

At the boundaries of the cavity, lateral thermal oxidation will occur between the silicon substrate and the bottom surface of the silicon nitride, resulting in a so-called bird's beak structure (Fig. 4). A typical height of the bird's beak structure, denoted as $t$ in the figure, ranges from 300 to $400 \mathrm{~nm}$. This lateral diffusion can be reduced using several schemes [21], however, the total height of the bird's beak is not a major concern in fabrication and performance.

A 500-nm LPCVD sacrificial phosphosilicate glass (PSG) is then blanket deposited. The wafer is annealed at $950^{\circ} \mathrm{C}$ for 1 $\mathrm{h}$. The PSG layer is then patterned using photolithography to define the sacrificial layer and the etching channels overlying etch cavity [Fig. 3(3)]. Unmasked PSG is etched away with buffered hydrofluoric acid within $20 \mathrm{~s}$.

Following the removal of the photoresist material, a 1.2$\mu \mathrm{m}$-thick low-stress silicon nitride is then deposited as the diaphragm material [Fig. 3(4)]. The silicon nitride material is selectively removed with SF6 plasma to expose the underlying sacrificial PSG. This plasma etch has a slow etch rate on the PSG layer, and, therefore, 20\% (time wise) overetch is done to ensure that all etch holes of the entire wafer area are clear.

In this stage, the sacrificial material is removed. Both sacrificial PSG and the thermal oxide are completely etched away using (49\%) hydrofluoric acid in 20 min. HF solution also etches silicon nitride, but at a very slow rate of approximately $40 \AA / \mathrm{min}$.

After etching, the wafer is thoroughly rinsed in deionized (DI) water for $1 \mathrm{~h}$ to purge $\mathrm{HF}$ from within the empty cavity through out diffusion. The water within cavities is then removed by spin drying the wafer at 7-krpm rotation speed; this is followed by convection-oven baking at $120^{\circ} \mathrm{C}$ for $1 \mathrm{~h}$.

A second LPCVD silicon-nitride layer (400 nm thick) is deposited at approximately $300 \mathrm{mTorr}(0.04 \mathrm{~Pa})$ and $850^{\circ} \mathrm{C}$ to seal the cavities under vacuum [23]. Because there are still water molecules inside the cavities after the baking, the deposition chamber is purged in nitrogen ambient at $600^{\circ} \mathrm{C}$ for 30 min before deposition starts; this step completely removes residue moisture before the high-vacuum nitride deposition 


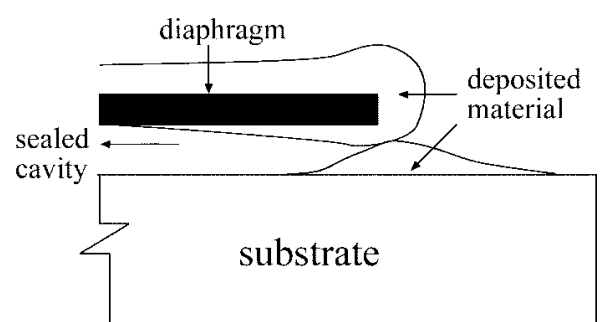

Fig. 5. A close-up cross-sectional view at the etch hole opening (A-A' cross section depicted in Fig. 2 of a sealed etching channel opening).

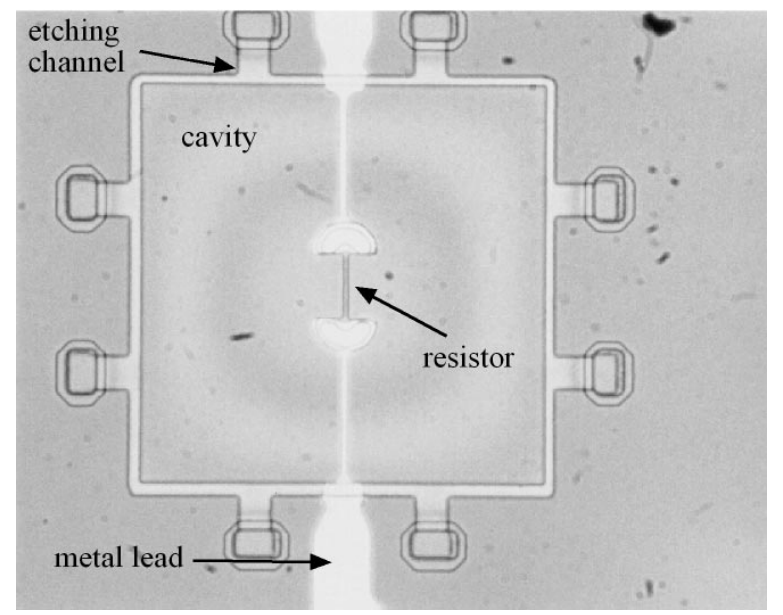

Fig. 6. Optical micrograph of a shear-stress sensor with a sealed cavity.

begins. During the LPCVD deposition, the deposition thickness is varied for different parts of the sensor. On top of the diaphragm there is an average deposition thickness; near the entrance of the etch channel, the deposition thickness is above average due to the increased space angle. The two deposition fronts eventually meet to permanently seal the cavity (Fig. 5).

To form the resistor, a 450-nm LPCVD polysilicon layer is deposited at $620^{\circ} \mathrm{C}$. The polysilicon film deposited at this temperature is completely crystallized [22] with crystal grain sizes on the order of $600 \AA$. Polysilicon doping is done by ion implantation with phosphorus using a total dose of $1 \times$ $10^{16} \mathrm{~cm}^{2}$ at $40 \mathrm{keV}$ of energy. The wafer is then annealed at $1000^{\circ} \mathrm{C}$ for $1 \mathrm{~h}$ to activate the dopant and reduce intrinsic stress in the as-deposited polysilicon material. The measured sheet resistivity of the polysilicon is $50 \Omega / \square$. The polysilicon is then patterned and plasma etched to form individual resistors. Following the removal of the photoresist, another 100-nm layer of LPCVD silicon nitride is deposited to passivate the polysilicon resistor. This film prevents resistance from longterm drifting due to spontaneous oxidation of the polysilicon resistor in air [24]. Contact holes are patterned and etched in plasma to allow for access to the polysilicon resistor through the last silicon nitride layer.

Finally, thermal evaporation produces the aluminum metallization (300 nm thick) which is patterned to form the leads. This thickness is found to be sufficient to ensure continuity of metal lines at the perimeter of the cavity.

Micrographs of the fabricated devices are shown in Fig. 6 . The cavity is $200 \times 200 \mu \mathrm{m}^{2}$, and the resistor is $40 \mu \mathrm{m}$ long

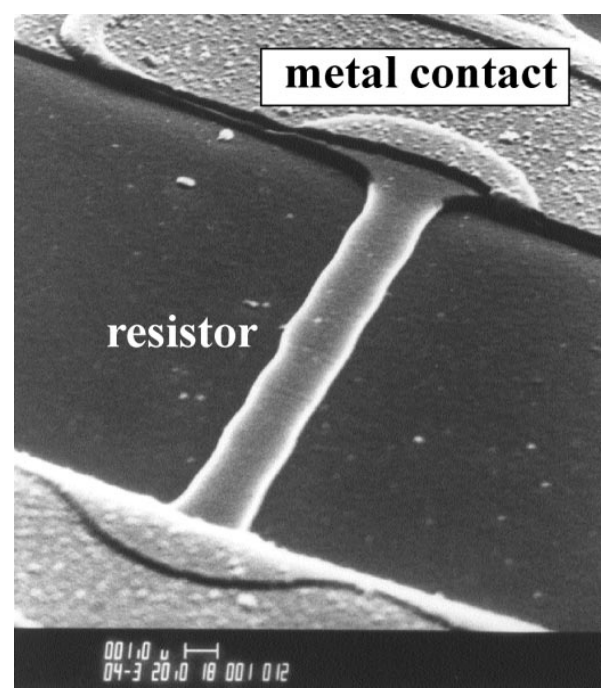

Fig. 7. A scanning electron micrograph of a polysilicon resistor.

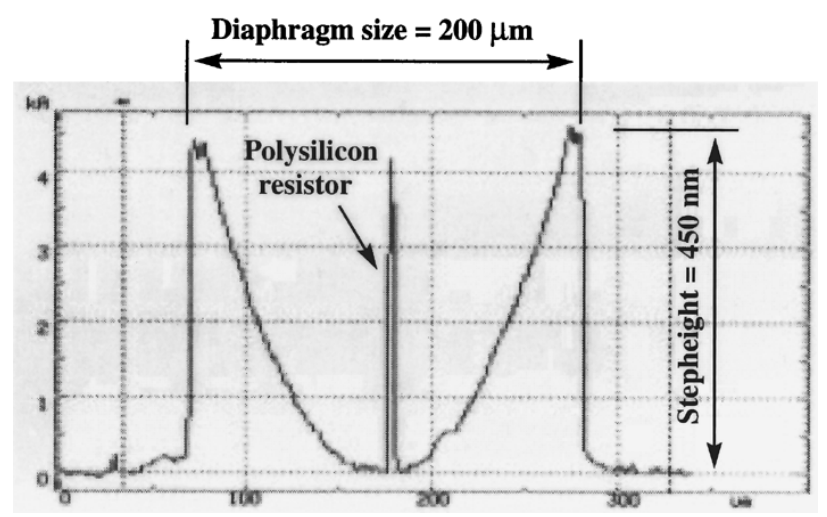

Fig. 8. Surface roughness profile across the sensor diaphragm and resistor.

and $2 \mu \mathrm{m}$ wide. Since the cavity is held under vacuum, the diaphragm is bent down by the external atmospheric pressure so that optical interference patterns (Newton rings) can be seen under the microscope. Fig. 7 is a scanning electron microscope graph of the polysilicon resistor.

It is important that the top surface of the shear-stress sensors be smooth so that the surface roughness will not cause unwanted flow fluctuation [2], [25]. Surface profiles of our sensors are examined using a surface profilometer (Tencor Instrument Alpha-Step 200) with a stylus force of $4 \mathrm{mg}$. Fig. 8 shows that even under simultaneous loading of both the stylus and the atmosphere pressure, the diaphragm has an overall roughness of only $450 \mathrm{~nm}$. In addition, this surface profile measurement also confirms that the diaphragm is not in contact with the cavity bottom under normal pressure conditions.

\section{SENSOR TESTINGS}

\section{A. Temperature Coefficient of Resistance}

We use a hot plate to heat up the sensor chip and measure the resistance at different temperatures in order to determine the temperature coefficient of resistance. The measured data of a typical resistor, which is $100 \mu \mathrm{m}$ long and $2 \mu \mathrm{m}$ wide, is 


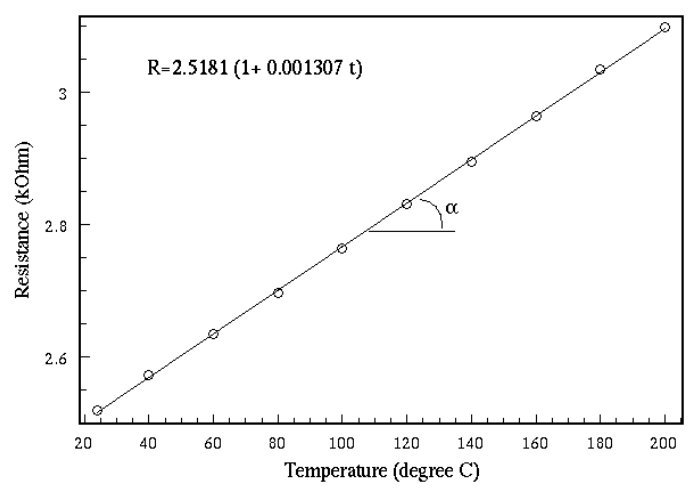

Fig. 9. The temperature coefficient of resistance (TCR) of a phosphorus-doped polysilicon resistor.

plotted in Fig. 9. Adequate time delay (20 min) is allowed between each temperature change to reach thermal equilibrium. The TCR of the heated resistor [see (2)], derived from the slope of linear curves, is $0.13 \% /{ }^{\circ} \mathrm{C}$. For comparison, the TCR of platinum and tungsten is $0.39 \%$ and 0.45 , respectively.

\section{B. Thermal Isolation}

The thermal isolation of vacuum-sealed cavities is evaluated next. This is demonstrated using a sensor that is $50 \mu \mathrm{m}$ long and $2 \mu \mathrm{m}$ wide with the nominal resistance being $1.25 \mathrm{k} \mu$. The thermal resistance $\theta$ is defined as a sensor element's temperature change $\triangle T$ due to an input power $P$ as in

$$
\triangle T=T-T_{0} \equiv \theta P .
$$

Plugging this expression into (2), the sensor resistance can be written as

$$
R=R_{0}(1+\theta P) .
$$

Three sensor structures are used for obtaining currentvoltage characteristics (Fig. 10). In the first case, the resistor sits on top of a standard vacuum-sealed cavity. In the second case, a crack is intentionally created in the diaphragm of the aforementioned sensor with the help of a micromanipulator probe, thus, the cavity becomes filled with air, which will increase the thermal conduction between the thermal element and the substrate. In the last case, a reference sensor element, identical with the first two resistors in geometry and resistance, is deposited directly on top of silicon substrate. Results of I-V measurements conducted in still air are shown in Fig. 11. The first resistor (located on the vacuum cavity) has the largest I-V-curve nonlinearity, proving that it experiences the most pronounced temperature rise due to ohmic heating among all three structures. On the other hand, the I-V curve of the resistor directly over the silicon substrate is almost linear, indicating that the thermal conduction to the substrate dominates the heat transfer process and little temperature variation has been created.

Using data from Fig. 11, resistance (V/I) is plotted as a function of input power (V.I) in Fig. 12. Furthermore, using the data from Fig. 12 and the known TCR of resistors, the increase of temperature can be deduced (Fig. 13). According to (5), the slopes of these three curves indicate the thermal

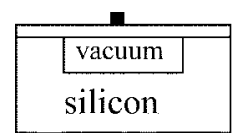

(a)

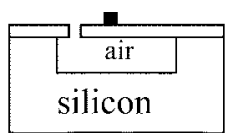

(b)

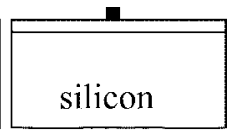

(c)
Fig. 10. Schematic diagrams of (a) a polysilicon resistor located over a vacuum cavity and (b) on top of an air-filled cavity and (c) an identical resistor directly on top of the substrate.

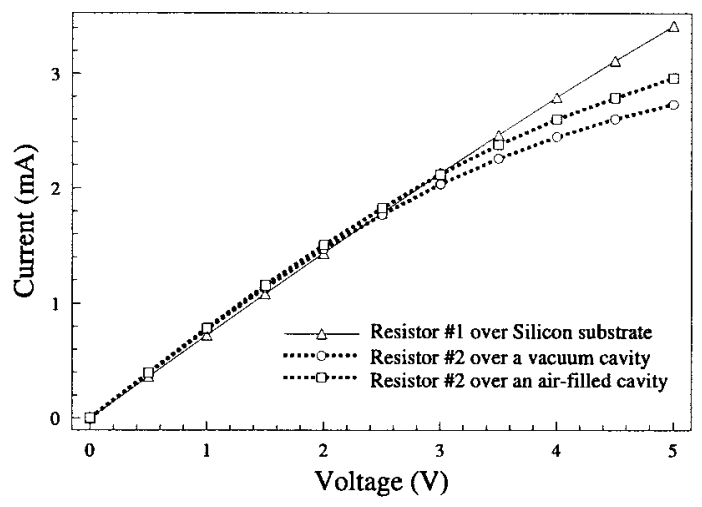

Fig. 11. I-V characteristics of three resistors in Fig. 10.

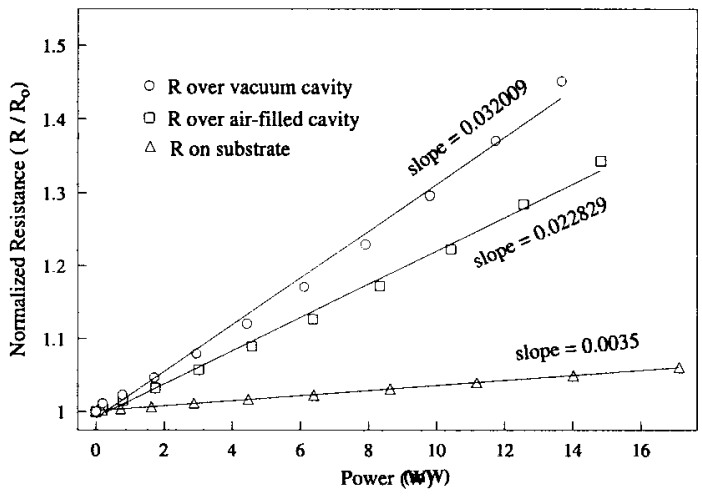

Fig. 12. Calculated sensor resistance at various power inputs.

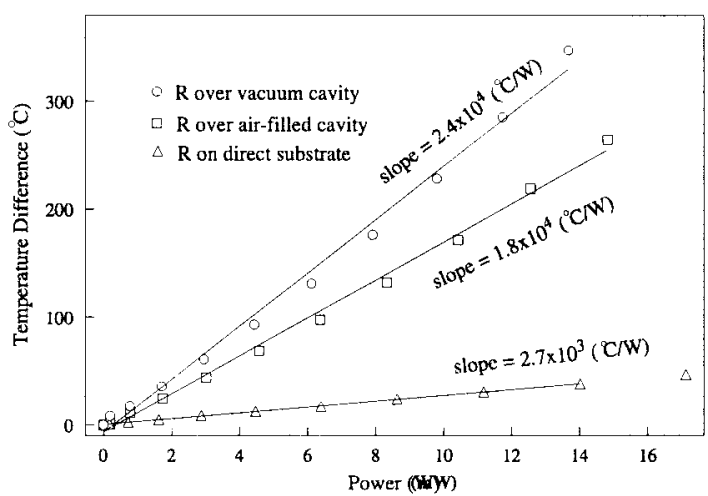

Fig. 13. Resistor surface temperature (derived from the previous figure) at various power inputs.

resistivity $\theta$. It is found that $\theta$ is $2.4 \times 10^{4 \circ} \mathrm{C} / \mathrm{W}$ for case 1 (resistor on a vacuum cavity), $1.8 \times 10^{4 \circ} \mathrm{C} / \mathrm{W}$ for case 2 (resistor over an air-filled cavity), and $2.7 \times 10^{3 \circ} \mathrm{C} / \mathrm{W}$ for the last case (resistor directly on the substrate). It is clear that the 


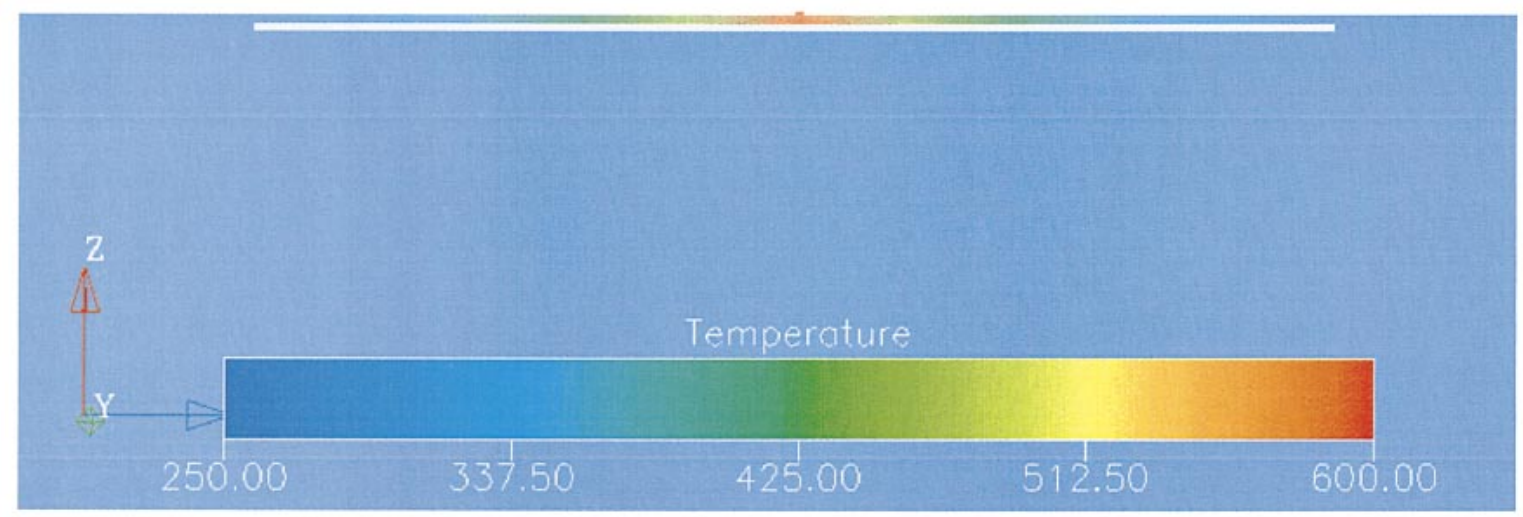

Fig. 14. Cross-sectional view of the temperature distribution of a shear-stress sensor with cavity. The power input is $14.4 \mathrm{~mW}$.

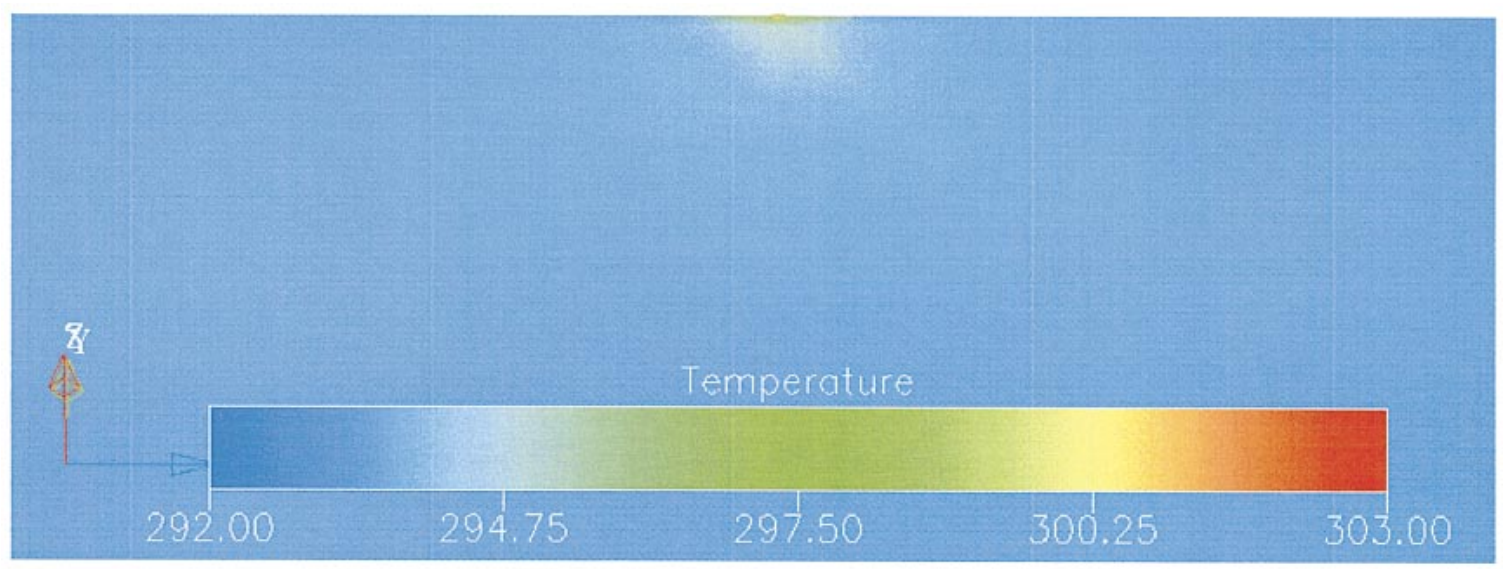

Fig. 15. Cross-sectional view of the temperature distribution of a shear-stress sensor without cavity (case 3). The input power is $14.4 \mathrm{~mW}$.

vacuum cavity improves the thermal isolation: $\theta$ for case 1 is 1.33 times and 9 times compared with that of cases 2 and 3 , respectively.

The analysis of thermal transfer for the first and third cases has been conducted using electrothermal numerical simulation packages (MEMCAD program by Microcosm Corporation, Cambridge, MA). The second case is difficult to simulate because the solid model cannot be built readily. The distribution of temperature for both cases is illustrated in Figs. 14 and 15. The distribution of temperature as a function of the input power is summarized in Fig. 16. The estimated temperature of the sensor resistor is lower than the measurement results. However, due to possible error related to mesh generation and parameter definition, the simulation result provide adequate substantiation to experimental data.

\section{Wind-Tunnel Setup}

An experimental wind tunnel with a maximum free-stream velocity of $30 \mathrm{~m} / \mathrm{s}$ is used for sensor characterization (Fig. 17). Its test section is $5 \mathrm{~m}$ long, $60 \mathrm{~cm}$ wide, and $2.5 \mathrm{~cm}(h)$ high. Since the width over height ratio of the test section is $60 / 2.5=24$, flow is assumed to be 2-D, i.e., flow pattern is independent of span-wise location.

The sensor package is $2 \times 2 \mathrm{~cm}^{2}$ in area, and the sensor surface is mounted flush with the wind-tunnel sidewall. The sensor package is located in the mid span on the top wall of

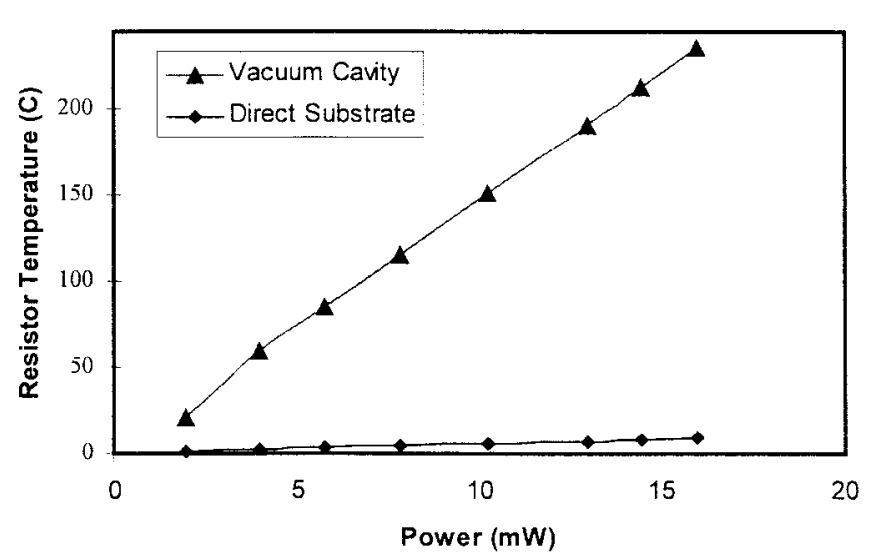

Fig. 16. Distribution of temperature as a function of input power obtained using numerical simulation.

the wind tunnel in a downstream region where turbulence flow is fully developed (for the velocity range between $10-30 \mathrm{~m} / \mathrm{s}$ ).

\section{Biasing of Sensors}

An important parameter for characterizing the operation condition of the shear-stress sensor is the over heat ratio. The temperature overheat ratio $\alpha_{T}$, defined as the relative change of sensor temperature compared to the ambient temperature, is

$$
\alpha_{T}=\left(T-T_{0}\right) / T_{0}
$$




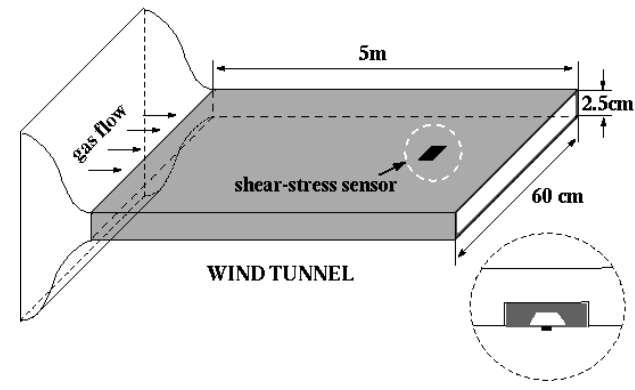

Fig. 17. Location of the shear-stress sensor within a wind tunnel.

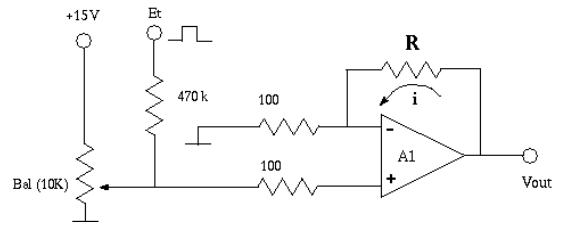

Fig. 18. Constant-current mode driving circuit.

It is also practical to define a resistance overheat ratio $\alpha_{R}$ as the relative change of sensor resistance compared to the resistance at the ambient temperature

$$
\alpha_{R}=\left(R-R_{0}\right) / R_{0} .
$$

The resistance overheat ratio is related to the temperature overheat ratio by

$$
\alpha_{R}=\alpha T_{0} \alpha_{T}
$$

The amount of current that can be used to bias the sensor is limited by two factors. First, the resistance overheat ratio is set to be less than 0.25 in order to minimize natural convection caused by the heating of the resistive element. The current density through the aluminum must be smaller than $10^{5} \mathrm{~A} / \mathrm{cm}^{2}$ in order to avoid long-term damage to sensor due to potential electromigration of aluminum.

Since the resistance of a micromachined hot-film sensor $(>1 \mathrm{k} \Omega)$ is generally higher than the value of a traditional hot-wire sensor (approximately $50 \Omega$ ), conventional driving circuits for anemometers need to be modified for present use. Three different types of circuits have been developed for our experiments [26]: constant current (CC), constant voltage $(\mathrm{CV})$, and constant temperature (CT). The CV circuit has the simplest configuration, and the CT circuit is the most complex. The CT circuit also provides the fastest time response among all three.

In both $\mathrm{CC}$ and $\mathrm{CT}$ circuits, pulsed signals are fed into the circuits via terminal $E_{t}$ for time-constant measurements. The constant-current circuit is shown in Fig. 18 in which $R$ represents the sensor resistor. $V_{\text {out }}$ is the output voltage. The CT circuit (Fig. 19) contains a feedback loop that keeps the voltage across the sensor resistor $\mathrm{R}$ constant. Resistors $\mathrm{R} 4$, $\mathrm{R} 6, \mathrm{R}$, and $\mathrm{Ra}$ form a bridge. Before a circuit is used to drive a sensor, we select an overheat ratio and balance the bridge. When the switch S1 is connected to the calibration terminals, the combination of R16 and R11 determines the overheat ratio. For example, when R16 is $27 \mathrm{k} \Omega$ and R11 is $220 \mathrm{k} \Omega$, the

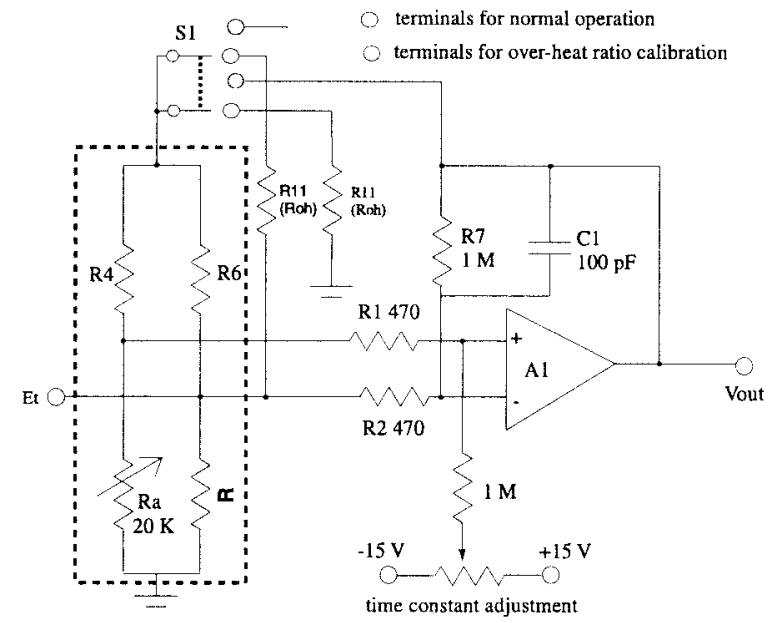

Fig. 19. Constant-temperature mode driving circuits.

overheat ratio is 0.1. After calibration, S1 is switched back to the terminals for normal operation. More details about the operation of the CT circuit can be found in [27].

The thermal noise of the resistor increases with temperature. The rms value of the output voltage due to the thermal noise is expressed as

$$
V=\sqrt{4 k R \triangle f T}
$$

where $k$ is the Boltzmann's constant and $\triangle f$ is the frequency bandwidth of the circuit. The noise level is expected to increase as the temperature of the sensor is raised further. The thermal noise of a $10-\Omega$ resistor at a temperature of $490 \mathrm{~K}$ and a bandwidth of $100 \mathrm{kHz}$ is $5.2 \mu \mathrm{V}$. We have not measured the noise experimentally, however, the above analysis indicates that the calculated noise level can be neglected compared to the signal level.

\section{E. Time Response}

Time constants of sensors are obtained experimentally. Since suitable velocity fluctuations are not readily available, one usually relies on electronic test signals. The time constant can be obtained by feeding an electronic sine wave or square wave into the $E_{t}$ terminal of both CC and CT circuits [9], [28]. Most commonly, when a step current (square wave) passes through the resistor, the transient voltage response is used to deduce the time constant. Using CC mode, the measured time constants of the microsensor are on the order of several hundred microseconds. For example, a resistor that is $100 \mu \mathrm{m}$ long and $2 \mu \mathrm{m}$ wide exhibits a thermal time constant of 350 $\mu$ s and a cutoff frequency of $1.9 \mathrm{kHz}$. Using CT circuitry with feedback control, the time constant is decreased drastically. The measured time constant is $72 \mu \mathrm{s}$, which is consistent with the results indicated by theoretical models [29]. According to the approximate relation between time constant $t_{c}$ and cutoff frequency $f_{c}$ for CT operation, $f_{c}=1 /\left(1.5 t_{c}\right)$; the cutoff frequency is estimated as $9 \mathrm{kHz}$. The time constant of the shear-stress sensor device in [10], in comparison, is $25 \mu \mathrm{s}$ under constant temperature operation. According to [29], the time constant $\tau$ is related to the thermal transfer through the 


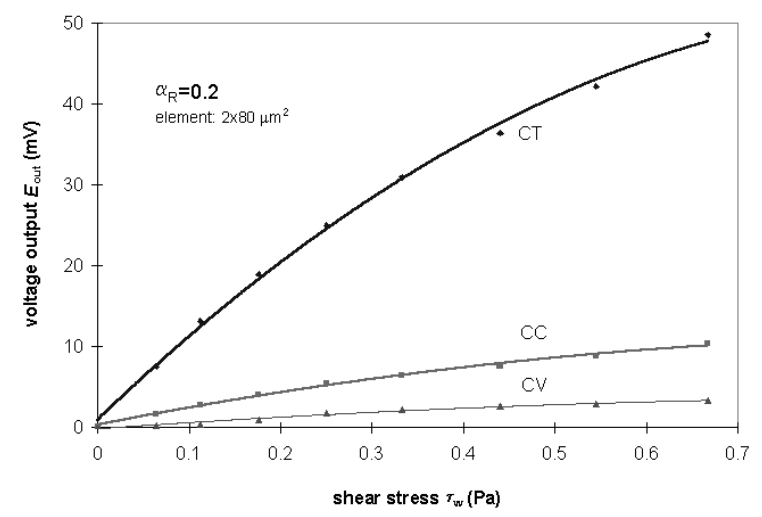

Fig. 20. Output voltage versus wall shear stress for CC, CV, and CT operation modes.

diaphragm material. Its magnitude can be estimated as

$$
\tau=\frac{c m}{4 t k}
$$

where $c$ is the specific heat, $m$ is the mass of the film, and $t$ and $k$ are the thickness and thermal conductivity of the supporting film, respectively. Assuming that the effective heated area is $1 \%$ of the nominal area of the diaphragm (Fig. 14), we have confirmed that the time constant calculated using the above equation has the same order of magnitude compared with the experimental data.

\section{F. Sensor Calibration}

Wind-tunnel calibration of the micromachined shear-stress sensors is conducted under $\mathrm{CC}, \mathrm{CV}$, and $\mathrm{CT}$ driving modes. The steady-state sensor voltage output (time averaged over a 2-min period) with respect to the mean-stream velocity is measured at various flow velocities ranging between $5-25 \mathrm{~m} / \mathrm{s}$.

The shear stress $(\tau)$ is related to the stream-wise pressure distribution in the wind tunnel by [30]

$$
\frac{d P_{x}}{d x}=-\frac{\tau_{w}}{h}
$$

where $P_{x}$ is the local pressure, $x$ is the stream-wise coordinate, and $h$ is the half height of the wind tunnel. We measure the stream-wise pressure gradient with the help of pressure taps along the sidewall of the wind tunnel. $\tau$ is calculated by using (11).

The calibration results for one sensor unit $\left(R_{0} \approx 2 \mathrm{k} \Omega\right)$ at the same resistive overheat ratio $\alpha_{R}$ of 0.2 , but different operation modes are shown in Fig. 20. The top curve, for CT mode operation, has a higher sensitivity compared with that of the CC and CV mode operations. Data shown in Fig. 21, with the heating power $P=i^{2} R$ divided by the temperature different $\triangle T$, are replotted as a function of the shear stress to the one thirds power-a linear relation is confirmed. According to (3), the slope of the curve corresponds to $A$ and the intercept on the $P / \triangle T$ axis is $B$. The fitting parameters for the three modes are summarized in Table I.

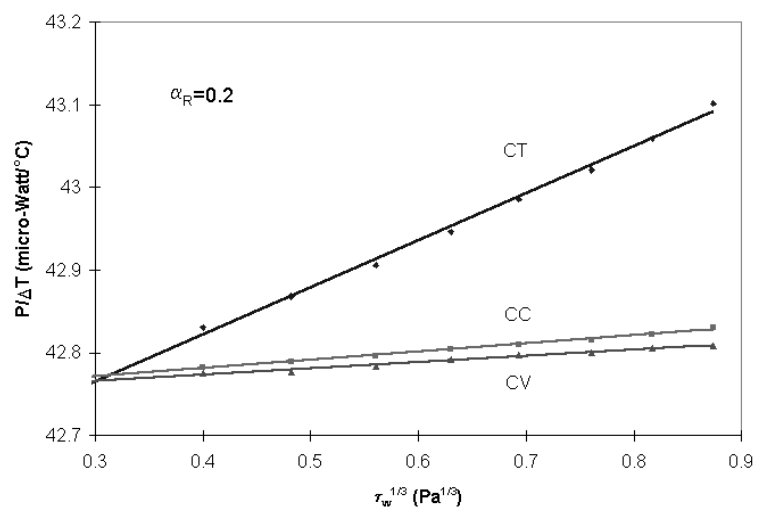

Fig. 21. The ratio between input power and temperature variation versus the shear stress to the $1 / 3$ power.

TABLE I

Fitting Parameters for Sensor OutPut Using CV, CC, And CT Modes

\begin{tabular}{ccc}
\hline mode & $\mathrm{A} \rho^{1 / 3}$ & $\mathrm{~B}$ \\
\hline constant voltage & 0.0755 & 42.7432 \\
constant current & 0.10015 & 42.7409 \\
constant temperature & 0.5698 & 42.593 \\
\hline
\end{tabular}

\section{G. Sensitivity to External Pressure}

The fabricated sensor is also sensitive to changes of ambient pressure since the heavily doped polysilicon serves as a piezoresistive element. The pressure sensitivity is relatively small compared with the sensitivity to shear stress and temperature. Under an applied positive pressure of $p$, the average longitudinal compressive strain $\left\langle\varepsilon_{l}\right\rangle$ on a polysilicon resistor (with a length of $40 \mu \mathrm{m}$ ) is approximately $\frac{0.15 p a_{2}}{\pi^{2} E t^{2}}$, with $a$ and $t$ being the width and thickness of the diaphragm, respectively [31]. Assuming the Young's modulus of the diaphragm $(E)$ is $160 \mathrm{GPa}$, the average strain is $\varepsilon_{l}=1.7 \times 10^{-9} p$. The relative change of resistance due to $p$ is $\frac{\Delta R}{R}=G \cdot \varepsilon_{l}=3.4 \times 10^{-8} p$, with $G=20$ being the gauge factor of the polysilicon resistor. If the pressure changes by one atmosphere, i.e., $10^{5} \mathrm{~Pa}$, $\frac{\triangle R}{R}$ equals $0.34 \%$. This is much smaller compared with the resistance overheat ratio under common operating conditions. The sensitivity with respect to pressure will increase with increasing length of the polysilicon resistor. As an example, if the length of the resistor reaches $100 \mu \mathrm{m}$ or $0.5 a,\left\langle\varepsilon_{l}\right\rangle=$ $\frac{0.2 p a^{2}}{\pi^{2} E t^{2}}$

\section{SUMmary}

A micromachined boundary-layer shear-stress sensor has been developed. The sensors operate on thermal-transfer principles-they are fabricated using surface micromachining techniques. The heat generating and sensing element, a polycrystalline silicon resistor, is located on top of a vacuumsealed cavity, and the heat loss from the resistor to the substrate is therefore reduced tremendously. Shear-stress calibration within a wind tunnel uses three different driving modes: CC, CV, and CT. The response curves agrees with theoretical analysis well. CT mode operations show the 
highest shear-stress sensitivity and the fastest frequency response.

\section{REFERENCES}

[1] J. H. Haritonidis, "The measurement of wall shear stress," Advances in Fluid Mechanics Measurements. New York: Springer-Verlag, pp. 229-261, 1989.

[2] R. J. Goldstein, Fluid Mechanics Measurements. New York: Hemisphere, p. 61, 1983.

[3] J. H. Preston, "The determination of turbulent skin friction by means of Pitot tubes," J. R. Aero. Soc., vol. 58, pp. 109-121, 1953.

[4] J. H. Hool, "Measurement of skin friction using surface tubes," Aircr. Eng., vol. 28, p. 52, 1956

[5] L. F. East, "Measurement of skin friction at low subsonic speeds by the razor-blade technique," $R \& M$ 3525, Aero. Res. Counc., London, U.K., 1966.

[6] M. A. Schmidt, R. T. Howe, S. D. Senturia, and J. H. Haritonidis, "Design and calibration of a microfabricated floating-element shearstress sensor," IEEE Trans. Electron. Devices, vol. 35, no. 6, pp. $750-757,1988$.

[7] J. Shajii, K. Y. Ng, and M. A. Schmidt, "A microfabricated floatingelement shear-stress sensor using wafer-bonding technology," IEEE J. Microelectromech. Syst., vol. 1, no. 2, pp. 89-94, 1992.

[8] A. Padmanabhan, H. Goldberg, K. D. Breuer, and M. Schmidt, "A wafer bonded floating element shear stress microsensor with optical position sensing by photodiodes," IEEE J. Microelectromech. Syst.., vol. 5, no. 4, pp. 307-315, 1996.

[9] T. J. Hanratty and J. A. Cambell, "Measurement of wall shear stress," Fluid Mechanics Measurements, R. J. Goldstein, Ed.. New York: Hemisphere, pp. 559-615, 1983.

[10] E. Kälvesten, C. Vieider, L. Löfdahl, and G. Stemme, "An integrated pressure-flow sensor for correlation measurements in turbulent gas flows," Sens. Actuators, vol. A52, pp. 51-58, 1996.

[11] B. W. van Oudheusden and J. H. Huijsing, "Integrated flow friction sensor," Sens. Actuators, vol. A15, pp. 135-144, 1988.

[12] B. W. van Oudheusden, "Silicon thermal flow sensors," Sens. Actuators, vol. A30, pp. 5-26, 1992.

[13] K. Petersen, J. Brown, and R. Legtenberg, "High-precision, highperformance mass flow sensor with integrated laminar flow microchannels," in Proc., 1985 Int. Conf. Solid-State Sensors and Actuators, pp. 361-363.

[14] Y. C. Tai and R. S. Muller, "Lightly-doped polysilicon bridge as a flow meter," Sens. Actuators, vol. 15, pp. 63-75, 1988.

[15] C. H. Mastrangelo, Master's thesis, Univ. Calif. Berkeley, CA, 1987.

[16] O. Tabata, "Fast-response silicon flow sensor with an on-chip fluid temperature sensing element," IEEE Trans. Electron Devices, vol. 33, pp. 361-365, 1986.

[17] B. W. van Oudheusden and A. W. van Herwaarden, "High sensitivity 2-D flow sensor with an etched thermal isolation structure," Sens. Actuators, vols. A21-23, pp. 425-430, 1990.

[18] L. Lofdahl, G. Stemme, and B. Johansson, "A sensor based on silicon technology for turbulence measurements," J. Phys. E: Sci. Instrum., vol. 22, pp. 391-393, 1989.

[19] G. N. Stemme, "A monolithic gas flow sensor with polyimide as thermal insulator," IEEE Trans. Electron Devices, vol. ED-33, p. 1470, 1986

[20] S. Wolf and R. N. Tauber, Silicon Processing for the VLSI Era, vol. 1. Sunset Beach, CA: Lattice, 1986.

[21] S. M. Sze, VLSI Technology, 2nd ed. New York: McGraw-Hill, 1988.

[22] E. Ibok and S. Garg, "A characterization of the effect of deposition temperature on polysilicon properties," J. Elec. Chem. Soc., vol. 140, no. 10 , pp. 2927-2937, Oct. 1993

[23] C. Liu and Y. C. Tai, "Studies on the sealing of micromachined cavities using chemical vapor deposition materials," in Technical Digest, SolidState Sensor and Actuator Workshop, Hilton Head Island, SC, 1994, pp. 103-106.

[24] K. C. Saraswat and H. Singh, "Thermal oxidation of heavily phosphorous-doped thin films of polycrystalline silicon," J. Electrochem. Soc., vol. 129, pp. 2321-2326, 1982.

[25] S. Tung, personal communication.

[26] J. B. Huang, C. Liu, F. K. Jiang, S. Tung, Y. C. Tai, and C. M. Ho, "Fluidic shear-stress measurement using surface-micromachined sensors," in IEEE 1995 Region 10 Conf. Microelectronics and VLSI, Hong Kong, Nov. 1995, pp. 570-574

[27] B. Gupta, R. Goodman, F. Jiang, and Y. C. Tai, "Analog VLSI system for active drag reduction," IEEE J. Microelectromech. Syst., vol. 16, no. 5, pp. 53-59, 1996.

[28] M. J. Moen and S. P. Schneider, "The effect of sensor size and substrate properties on the performance of flush-mounted hot-film sensors," Thermal Anemometry-1993, D. E. Stock, S. A. Sherif, A. J. Smits, and J. Davidson, Eds. ASME, pp. 249-261, 1993.

[29] J. B. Huang, C. M. Ho, S. Tung, C. Liu, and Y. C. Tai, "Micro thermal shear stress sensor with and without cavity underneath," in IEEE Instrumentation/Measurement Tech. Conf., IMTC'95, Waltham, MA, Apr. 1995, pp. 171-174.

[30] A. K. Hussain and W. C. Reynolds, "The mechanics of a perturbation wave in turbulent shear flow," AFOSR Scientific Rep. 177SR-1655TR.

[31] J. Q. Liu, "Integrated micro devices for small scale gaseous flow study," Ph.D. dissertation, Calif. Instit. Technol., Pasadena, 1995.

Chang Liu (M'91), for a biography, see this issue, p. 17.

Jin-Biao Huang received the B.Sc., M.Sc., and Ph.D. degrees in electrical engineering from Nanjing Institute of Technology (now Southeast University), Nanjing, China, in 1983, 1986, and 1989, respectively.

After completing his education, he joined the Microelectronics Center, Southeast University, where he was an Associate Professor from 1982 to 1993. In 1993, he joined the Center for Micro Systems at the University of California, Los Angeles. He conducted research in the area of sensors, MEMS, electronics measurements, and fluid mechanics. He is currently a Visiting Scientist at the Cornell Nanofabrication Facility and working for Boonton Electronics Corporation through the New Jersey Institute of Technology.

Zhenjun (Alex) Zhu received the B.S. degree from Tsinghua University, Beijing, China.

He is currently a Research Assistant Student at the Microelectroincs Laboratory, University of Illinois, Urbana. Currently, his research interests include microfabrication technologies and MEMS. He is interested in computer-aided design of MEMS. He developed an anisotropic crystalline etching simulation program.

Fukang Jiang received the B.S. degree from Hangzhou University, Hangzhou, China, in 1994 and the M.S. and Ph.D. degrees from the California Institute of Technology, Pasadena, in 1992 and 1998, respectively.

$\mathrm{He}$ is currently a Post-Doctoral Research Associate at the Micromachining Laboratory, California Institute of Technology. His current research includes the development of MEMS flow sensors and flexible skins with integrated sensors and electronics.

Steve Tung received the B.S. degree in mechanical engineering from the National Taiwan University, Taiwan, R.O.C., in 1984 and the Ph.D. degree from the University of Houston, Houston, TX, in 1992.

He became a Lecturer in the Department of Mechanical Engineering, University of Houston. In 1993, he joined the Center for Micro Systems, University of California, Los Angeles, as a Research Associate. There, he conducted research in the area of active drag reduction through the implementation of MEMS technology. His research interests include turbulence control, micromachine-based sensors and actuators, aerodynamics, aeroacoustics, and multiphase flows. 


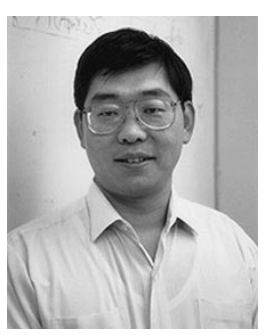

Yu-Chong Tai received the B.S. degree in electrical engineering from the National Taiwan University, Taipei, Taiwan, R.O.C., in 1981 and the M.S. and Ph.D. degrees from the University of California, Berkeley, in 1986 and 1989, respectively.

$\mathrm{He}$ is currently an Associate Professor of Electrical Engineering, California Institute of Technology, Pasadena, where he directs the Caltech Micromachining Laboratory, which currently sponsors more than 20 researchers for micromachining. He has over 12 years of experience in micromachines and/or MEMS research. His research interests include MEMS technology, microsensors, microactuators, microstructures, MEMS systems, and MEMS science. He successfully developed MEMS devices in his lab including pressure sensors, shear-stress sensors, hot-wire anemometers, magnetic actuators, microphones, microvalves, micromotors, etc. His system-level MEMS research projects include integrated M3 (microelectroincs + microsensors + microactuators) drag-reduction smart surface, flexible smart skin for the control of unmanned aerial vehicles, and microfluid delivery systems. He is also interested in MEMS sciences such as MEMS material (mechanical and thermal) properties, microfluid mechanics, and micro/nanoprocessing issues.

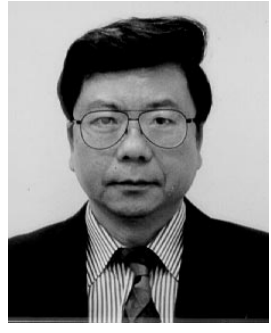

Chih-Ming Ho received the B.S. degree from the National Taiwan University, Taipei, Taiwan, R.O.C., and the Ph.D. degree in mechanics from Johns Hopkins University, Baltimore, MD.

He is the Ben Rich-Lockheed Martin Professor of the Mechanical and Aerospace Engineering Department, University of California, Los Angeles, and is the Director of the Center for Micro Systems. He was a Guest Editorial Committee Member of the Annual Review of Fluid Mechanics in 1995-96. He was an Associate Editor of the ASME Journal of Fluids Engineering from 1990 to 1993 and was an Associate Editor of the AIAA Journal from 1985 to 1987.

Dr. Ho was elected as a Member of the National Academy of Engineering in 1997 and the Academia Sinica in 1998. He was elected as a Fellow of the American Institute of Aeronautics and Astronautics for his seminal contributions to the basic understanding and control of turbulent shear flows and for pioneering contributions to applying microtransducers to aerospace science. He is also a Fellow of the American Physical Society. He was the Chair of Fluid Dynamics Division of APS in 1995-96. 